INTERNATIONAL JOURNAL OF RESEARCHES IN BIOSCIENCES, AGRICULTURE AND TECHNOLOGY (c) VISHWASHANTI MULTIPURPOSE SOCIETY (Global Peace Multipurpose Society) R. No. MH-659/13(N) www.vmsindia.org

\title{
A RARE SYNNEMATAOUS FUNGI FROM GHATANJI, (MS) INDIA.
}

\section{A. Shahezad and N. Dharkar}

S.P.M. Science and Gilani Arts Commerce College Ghatanji, Dist.Yavatmal (MS) India shahezadakil@yahoo.com

\section{Abstract:}

Present paper deals with a rare synnematous fungi from Ghatanji. FungiPodosporium is having synnamatatious conidiomata, conidia are slender, elongate, multiseptate, obclavate.

Keywords :- Synnematou fungi, conidia multiseptate, clavate, Podosporium new species.

\section{Introduction:}

During routine mycological survey of Ghatanji forest and near by area the author has collected a rare synnematou fungus viz Podosporium and on detailed morphological study, the specimen in question is treated as new species Podosporium megasporium sp.nov.

\section{Materials and Method:}

The collected specimens were wrapped in butter paper and bagged in envelope. By taking hand sections, semi permanent microscopic slides were prepared by using cotton blue as stain. Sections of the material were studied with the help of relevant keys and literature (Ainsworth et al 1973, , Barnett and hunter 1972, Jamaluddinet al 2004,). The specimen were deposited in Ajrekar Mycological Herbarium, Agharkar Research Institute (ARI) Pune 411004.

Podosporium megasporium sp.nov. (Plate 1, Fig.-1 a,b,c,d) (Etymology: After large size of spore)
Colonies effuse, brown, synnemata long straight measure $3.192-4.522 \mathrm{~mm}$ in length; conidiophores synnematous, branched, brown, smooth septate measure $13.2-33.0 \mu \mathrm{m}$ in diam; conidiogenous cells produce blastospores monoteric, terminal to intercalary, clavate, conidia solitary dry,apical simple obclavate, brown, 5-9septate measure 62.7-227.7x19.8$33 \mu \mathrm{m}$.

Collonies effuse brunne synnematae brunnea longae rigidea magnit 3.192-4.522 mm ; conidiophore synnematous, nonramosa, brunnea, leviter, septata magnit13.2-33.0 $\mu \mathrm{m}$ in diametro; cellulae conidiogenae blastosporae, productae, monotreatae, terminaliter vel integrator, clavatae; conidia solitoria, aridi simlicibus clavatae, brunnae 5-9 septatae magnit62.7-227.7x19.8-33 $\mu \mathrm{m}$.

Matrix:On dead stem of Azadirachta indica Juss legit MAS at BeloraGhatanji on 3/10/2003.No.AMH 9138.

\section{Comparison between Podosporium species}

\begin{tabular}{|l|l|l|l|l|}
\hline Species & Synnemata & Conidiophore & Conidia & Reference \\
\hline $\begin{array}{l}\text { P.viticola } \\
\text { Munjal\&Kapoor }\end{array}$ & $1.5 \mathrm{mmin}$ length & $4-8 \mu \mathrm{m}$ broad & $\begin{array}{l}60-92 \times 16- \\
20 \mu \mathrm{m}\end{array}$ & $\begin{array}{l}\text { Munjal\&Kapoor } \\
(1963)\end{array}$ \\
\hline $\begin{array}{l}\text { P.furcatum } \\
\text { Sharma \&Panwar }\end{array}$ & $1-2.5 \mathrm{~mm}$ long & $3-5 \mu \mathrm{m}$ thick & $\begin{array}{l}76-160 \mathrm{x} 12- \\
23 \mu \mathrm{m}\end{array}$ & $\begin{array}{l}\text { Sharma \& Panwar } \\
(1986)\end{array}$ \\
\hline $\begin{array}{l}\text { P.longatum } \\
\text { Chen\&Tzean }\end{array}$ & $\begin{array}{l}508-1102 \times 134- \\
196 \mu \mathrm{m}\end{array}$ & $\begin{array}{l}5.6-19.1 \times 3.5- \\
5.2 \mu \mathrm{m}\end{array}$ & $\begin{array}{l}62-118 \times 6.2- \\
10.3 \mu \mathrm{m}\end{array}$ & $\begin{array}{l}\text { Chen\&Tzean } \\
(1993)\end{array}$ \\
\hline $\begin{array}{l}\text { P.compactum } \\
\text { Teng }\end{array}$ & $\begin{array}{l}\text { 03-1.2mmhigh } \\
15-40 \mu \mathrm{m} \text { thick } \\
\text { base }\end{array}$ & $3-4 \mu \mathrm{m}$ thick & $25-58 \times 8-12 \mu \mathrm{m}$ & $\begin{array}{l}\text { Teng } \\
(1996)\end{array}$ \\
\hline $\begin{array}{l}\text { P.megasporium } \\
\text { sp.nov }\end{array}$ & $\begin{array}{l}3.192-4.522 \mu \mathrm{m} \text { in } \\
\text { length }\end{array}$ & $\begin{array}{l}13.2-33.0 \mu \mathrm{m} \\
\text { long }\end{array}$ & $\begin{array}{l}62.7- \\
227.7 \times 19.8- \\
33.0 \mu \mathrm{m}\end{array}$ & Understudy \\
\hline
\end{tabular}

Detailed morphological study and comparative study of known species the size of conidia is larger, hence, treated as new species. 


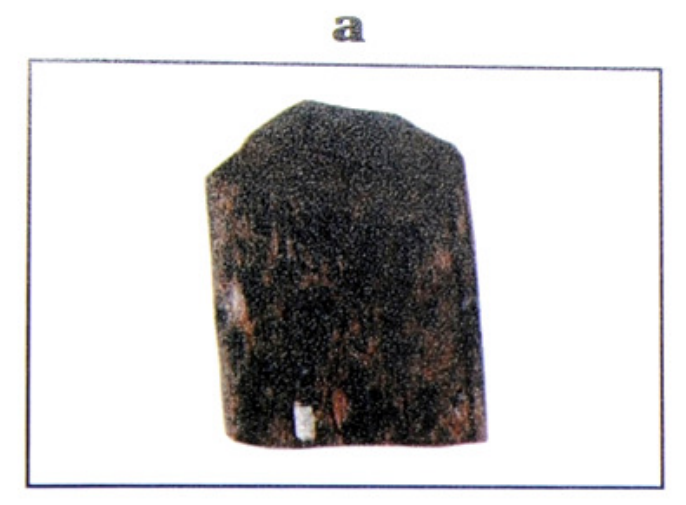

C

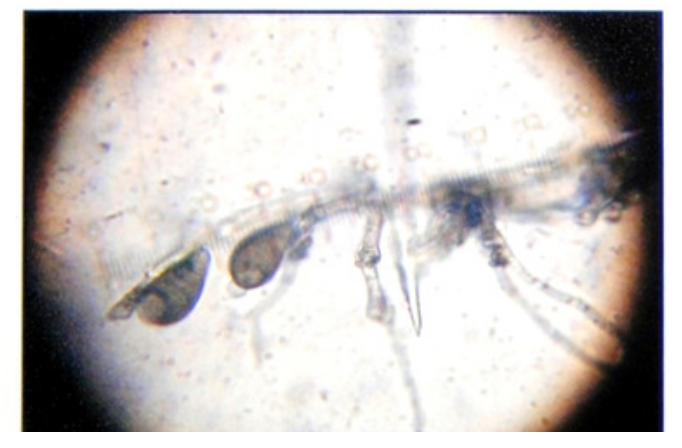

b

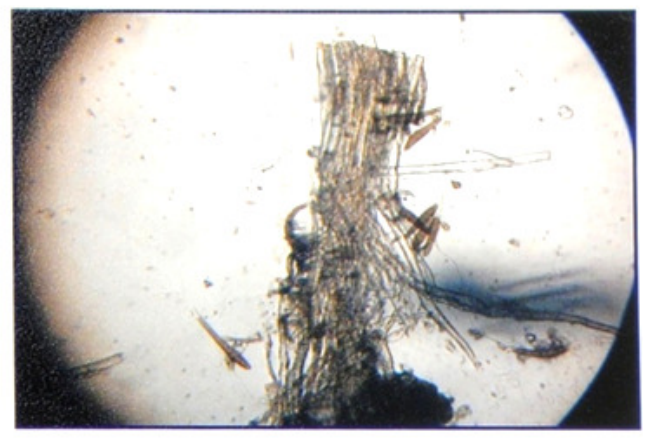

d

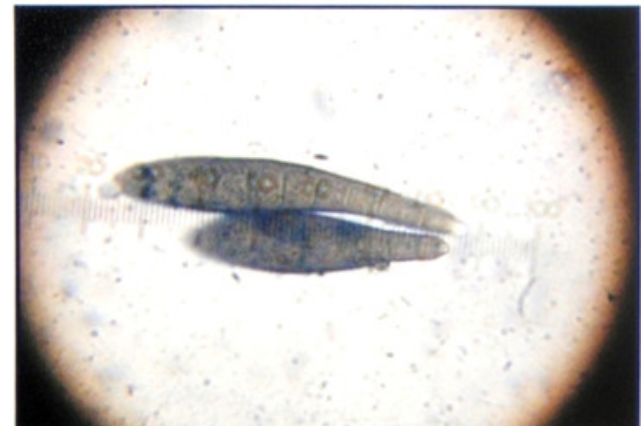

Figure. 1- Podosporium megasporium sp.nov.

$\mathrm{a}=$ Habit $\mathrm{b}=$ Synnema and Conidia, $\mathrm{c}=$ conidiogenous cell with Conidia $\mathrm{d}=$ Conidia $(45 \mathrm{x})$

\section{Acknowledgments:}

Authors are thankful to Dr. Anand Subhedar for encouragement and guidance and to S.A Gilani for providing research facilities.

\section{References:}

Ainsworth, G.C, Sparrow, F. K and Sussman, A.S. (1973): The fungi an advanced Treatise VOL IV A taxonomic review with keys; Ascomycetes and fungi ImperfectiAcademic press New York .Pp.621.

Barnett, H. L. \& B. B. Hanter (1972) : Illustrated Genera of imperfect fungi. III Ed, BurgossPublishing Co. Minnesota. Pp.240
Chen and Tzean(1993):Anamorphic fungi Podosporium elongatum. Mycol. Res.97:Pp637640.

Jamaluddin, S. Goswami, M.G and Ojha, B.M. (2004): Fungi of India (1989-2001) scientific publisher (India) Jodhpur. Pp. 326.

Munjal, R.L. and Kapoor, J.N. (1963) :Hyphomycetes from Himalaya. Indianphytopath. vol.16: Pp.86-93.

Sharma R. and Panwar, K.S. (1986): A new species of Podosporium. Curr.Sci.55:21:10911092.

Teng, S.C. (1996): Fungi of China. Mycotaxon LTD. Ithaca. New York. 